This is an electronic reprint of the original article. This reprint may differ from the original in pagination and typographic detail.

\author{
Author(s): Petrache, C. M.; Lv, B. F.; Astier, A.; Dupont, E.; Wang, Y. K.; Zhang, S. Q.; Zhao, P. W.; \\ Ren, Z. X.; Meng, J.; Greenlees, Paul; Badran, Hussam; Cox, Daniel; Grahn, Tuomas; \\ Julin, Rauno; Juutinen, Sakari; Konki, Joonas; Pakarinen, Janne; Papadakis, Philippos; \\ Partanen, Jari; Rahkila, Panu; Sandzelius, Mikael; Sarén, Jan; Scholey, Catherine; Sorri, \\ Juha; Stolze, Sanna; Uusitalo, Juha; Cederwall, B.; Aktas, Ö.; Ertoprak, A.; Liu, H.;
}

Title: $\quad$ Evidence of chiral bands in even-even nuclei

Year: $\quad 2018$

Version:

Please cite the original version:

Petrache, C. M., Lv, B. F., Astier, A., Dupont, E., Wang, Y. K., Zhang, S. Q., Zhao, P. W., Ren, Z. X., Meng, J., Greenlees, P., Badran, H., Cox, D., Grahn, T., Julin, R., Juutinen, S., Konki, J., Pakarinen, J., Papadakis, P., Partanen, J., . . A Andreoiu, C. (2018). Evidence of chiral bands in even-even nuclei. Physical Review C, 97(4), Article 041304(R). https://doi.org/10.1103/PhysRevC.97.041304

All material supplied via JYX is protected by copyright and other intellectual property rights, and duplication or sale of all or part of any of the repository collections is not permitted, except that material may be duplicated by you for your research use or educational purposes in electronic or print form. You must obtain permission for any other use. Electronic or print copies may not be offered, whether for sale or otherwise to anyone who is not an authorised user. 


\title{
Evidence of chiral bands in even-even nuclei
}

\author{
C. M. Petrache, ${ }^{1}$ B. F. Lv, ${ }^{1}$ A. Astier, ${ }^{1}$ E. Dupont,${ }^{1}$ Y. K. Wang, ${ }^{2}$ S. Q. Zhang, ${ }^{2}$ P. W. Zhao, ${ }^{2}$ Z. X. Ren, ${ }^{2}$ J. Meng, ${ }^{2,3}$ \\ P. T. Greenlees ${ }^{4}$ H. Badran, ${ }^{4}$ D. M. Cox,${ }^{4}$ T. Grahn, ${ }^{4}$ R. Julin, ${ }^{4}$ S. Juutinen, ${ }^{4}$ J. Konki,${ }^{4}$ J. Pakarinen,${ }^{4}$ P. Papadakis ${ }^{4}$ \\ J. Partanen, ${ }^{4}$ P. Rahkila, ${ }^{4}$ M. Sandzelius, ${ }^{4}$ J. Saren, ${ }^{4}$ C. Scholey, ${ }^{4}$ J. Sorri, ${ }^{4}$ S. Stolze,${ }^{4}$ J. Uusitalo, ${ }^{4}$ B. Cederwall, ${ }^{5}$ Ö. Aktas,${ }^{5}$ \\ A. Ertoprak, ${ }^{5}$ H. Liu, ${ }^{5}$ S. Matta, ${ }^{5}$ P. Subramaniam, ${ }^{5}$ S. Guo, ${ }^{6}$ M. L. Liu, ${ }^{6}$ X. H. Zhou, ${ }^{6}$ K. L. Wang, ${ }^{6}$ I. Kuti, ${ }^{7}$ J. Timár, \\ A. Tucholski, ${ }^{8}$ J. Srebrny, ${ }^{8}$ and C. Andreoiu ${ }^{9}$ \\ ${ }^{1}$ Centre de Sciences Nucléaires et Sciences de la Matière, CNRS/IN2P3, Université Paris-Saclay, Bât. 104-108, 91405 Orsay, France \\ ${ }^{2}$ State Key Laboratory of Nuclear Physics and Technology, School of Physics, Peking University, Beijing 100871, China \\ ${ }^{3}$ Yukawa Institute for Theoretical Physics, Kyoto University, Kyoto 606-8502, Japan \\ ${ }^{4}$ Department of Physics, University of Jyvaskyla, Jyvaskyla FIN-40014, Finland \\ ${ }^{5}$ KTH Department of Physics, S-10691 Stockholm, Sweden \\ ${ }^{6}$ Institute of Modern Physics, Chinese Academy of Sciences, Lanzhou 730000, China \\ ${ }^{7}$ Institute of Nuclear Research, Hungarian Academy of Sciences, 4001 Debrecen, Hungary \\ ${ }^{8}$ University of Warsaw, Heavy Ion Laboratory, Pasteura 5a, 02-093 Warsaw, Poland \\ ${ }^{9}$ Department of Chemistry, Simon Fraser University, Burnaby, BC V5A 1S6, Canada
}

(Received 13 December 2017; revised manuscript received 5 February 2018; published 27 April 2018)

\begin{abstract}
Evidence for chiral doublet bands has been observed for the first time in the even-even nucleus ${ }^{136} \mathrm{Nd}$. One chiral band was firmly established. Four other candidates for chiral bands were also identified, which can contribute to the realization of the multiple pairs of chiral doublet bands $(M \chi D)$ phenomenon. The observed bands are investigated by the constrained and tilted axis cranking covariant density functional theory (TAC-CDFT). Possible configurations have been explored. The experimental energy spectra, angular momenta, and $B(M 1) / B(E 2)$ values for the assigned configurations are globally reproduced by TAC-CDFT. Calculated results support the chiral interpretation of the observed bands, which correspond to shapes with maximum triaxiality induced by different multiquasiparticle configurations in ${ }^{136} \mathrm{Nd}$.
\end{abstract}

DOI: 10.1103/PhysRevC.97.041304

Chirality in nuclei is a dynamic collective mode that may appear in a triaxial nucleus which rotates about an axis out of the three principal planes of the ellipsoidal nuclear shape [1]. It gives rise to nearly degenerate bands which get closer in energy at high spin, when the collective angular momentum of the core directed along the intermediate axis is comparable to the angular momenta of the active quasiparticles directed along the short and long axes, and therefore the total angular momentum has a direction out of the three principal planes of the triaxial nucleus (3D direction) [1-4]. The chiral motion evolves from chiral vibration at low spins in which the rotation axis oscillates between the two 3D directions, to tunneling between wellestablished left-handed and right-handed configurations at high spins which corresponds to static chirality [5]. The simplest chiral geometry is realized in an odd-odd nucleus, with the angular momenta of the odd proton and odd neutron aligned along perpendicular axes, in a plane perpendicular to the angular momentum of the core. Such a 3D geometry of the angular momenta has been identified in odd-odd nuclei of the $A \sim$ 80, $A \sim 100, A \sim 130$, and $A \sim 190$ mass regions [6-16]. Nearly 3D rotation was also observed in odd-even nuclei, based on configurations involving three quasiparticles [17]. Significant progress for our understanding of chiral rotation is the possibility of having multiple pairs of chiral doublet bands (abbreviated as $\mathrm{M} \chi \mathrm{D}$ ) in a single nucleus as demonstrated theoretically in Refs. [18-22]. Strong experimental evidence for the existence of $\mathrm{M} \chi \mathrm{D}$ has been reported in the nuclei ${ }^{78} \mathrm{Br},{ }^{103} \mathrm{Rh}$, and ${ }^{133} \mathrm{Ce}[7,23,24]$. No chiral doublets were identified until now in even-even nuclei, even if nothing is against the existence of bands built on 4-quasiparticle (4-qp) configurations involving 2-qp aligned along one axis and 2-quasiholes (2-qh) aligned along a perpendicular axis. A tentative interpretation of a couple of 4-qp bands in ${ }^{136} \mathrm{Nd}$ in terms of chiral partners was proposed [25], but it was later questioned due to different reduced transition probabilities measured in the partner bands [26]. Candidates for chiral vibration have been reported in ${ }^{110,112} \mathrm{Ru}$ nuclei, but in that case the chiral geometry was not clearly revealed due to the shallow minima and low spins aligned along the short and long axes [27].

The present study was undertaken with the aim of searching for chiral partners in ${ }^{136} \mathrm{Nd}$, a nucleus in which the highspin states were investigated in several previous experiments $[25,26,28-33]$. In the present paper we only discuss the newly identified doublet bands in ${ }^{136} \mathrm{Nd}$. The very rich and complete level scheme established up to very high spins involving new normal deformed and highly deformed bands will be published in a forthcoming paper [34]. High-spin states in ${ }^{136} \mathrm{Nd}$ were populated using the ${ }^{100} \mathrm{Mo}\left({ }^{40} \mathrm{Ar}, 4 n\right)$ reaction and a $152-\mathrm{MeV}$ beam of ${ }^{40} \mathrm{Ar}$, provided by the K130 Cyclotron at the University of Jyväskylä, Finland. The target consisted of a self-supporting enriched ${ }^{100} \mathrm{Mo}$ foil of $0.5 \mathrm{mg} / \mathrm{cm}^{2}$ thickness. 


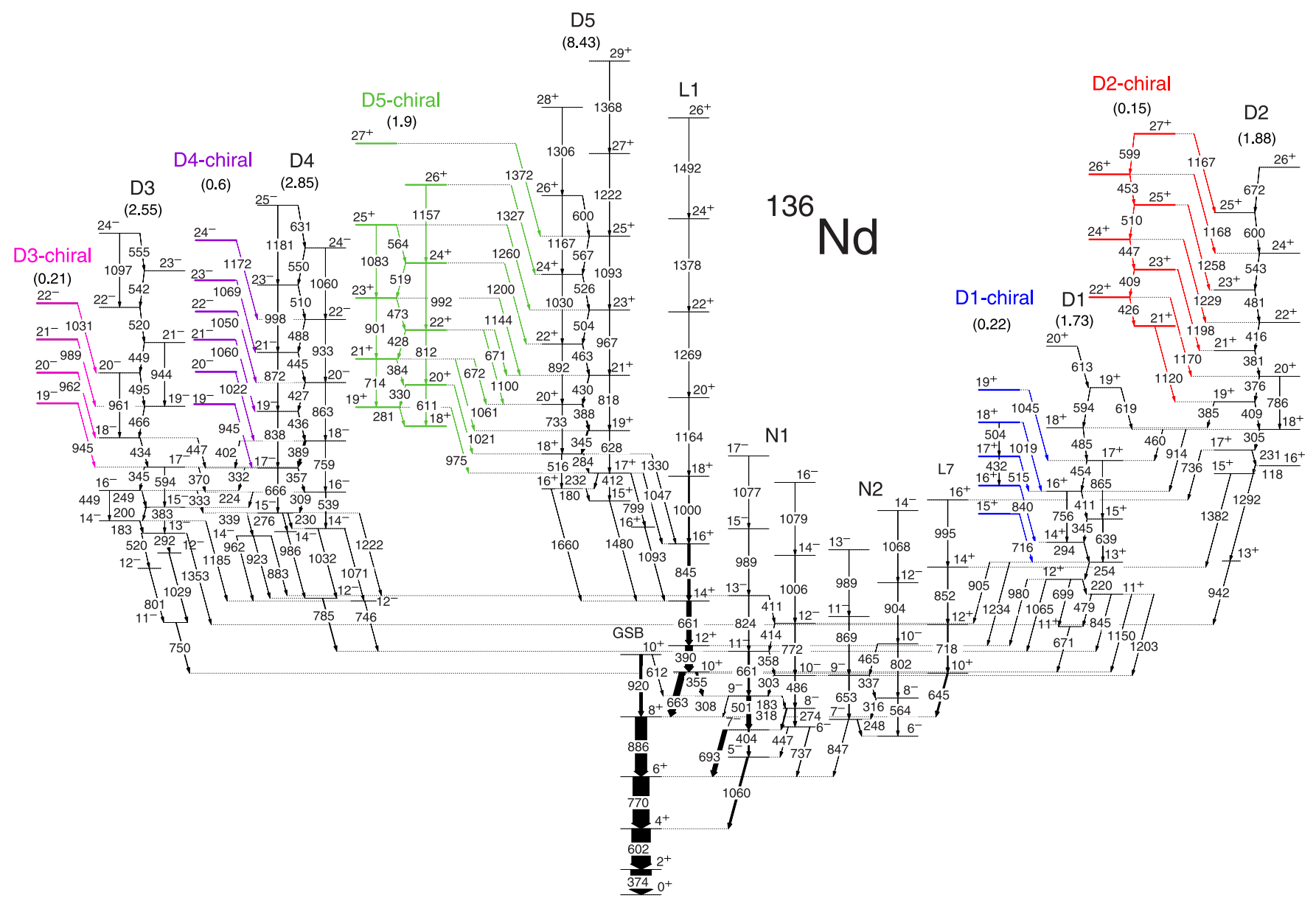

FIG. 1. Partial level scheme of ${ }^{136} \mathrm{Nd}$ showing the newly identified doublet bands and their decay toward low-lying levels. The intensities of the bands relative to the intensity of the $374-\mathrm{keV}, 2^{+} \rightarrow 0^{+}$transition are also indicated below the band labels. The bands are labeled as in the complete level scheme that will be published in a forthcoming paper [34].

The ${ }^{135} \mathrm{Nd}$ and ${ }^{136} \mathrm{Nd}$ nuclei were the most strongly populated in the reaction, with cross sections of around $100 \mathrm{mb}$ each, for a total of $480 \mathrm{mb}$. A number of $5.1 \times 10^{10}$ three-fold and higher prompt $\gamma$-ray coincidence events were accumulated using the JUROGAM II array which has an efficiency of around $4.3 \%$ at $1.3 \mathrm{MeV}$. The events were time stamped by the total data readout (TDR) data acquisition, and sorted using the GRAIN code [35]. Fully symmetrized, three-dimensional $\left(E_{\gamma}-E_{\gamma}-E_{\gamma}\right)$ and four-dimensional $\left(E_{\gamma}-E_{\gamma}-E_{\gamma}-E_{\gamma}\right)$ matrices of $\gamma$-ray energies $E_{\gamma}$ were analyzed using the RADWARE [36,37] analysis package. Spin and parity assignments were made on the basis of the measured DCO (directional correlations of oriented states) ratios $\left(R_{\mathrm{DCO}}\right)$, two-point angular correlation (anisotropy) ratio $R_{\mathrm{ac}}[38,39]$, angular distributions [40], and polarization asymmetries [41] of the transitions depopulating the states. Details of the angular correlation analysis will be given in a forthcoming paper [34].

The partial level scheme of ${ }^{136} \mathrm{Nd}$ showing five pairs of doublet bands and their decay toward low-lying states is given in Fig. 1. An example of double-gated spectra showing the transitions connecting one of the newly identified bands, band D2-C, to band D2, is given in Fig. 2. Similar spectra have been also obtained for the other doublet bands. One of the specific features of these bands is their decay to the yrast partners via high-energy quadrupole transitions, which, due to the $E_{\gamma}^{5}$ dependence, are stronger that the in-band dipole transitions which have low energy and $E_{\gamma}^{3}$ dependence, resulting in a factor of 10 to 20 in favor of the connecting transitions. Due to this fact, many in-band dipole transitions of the new bands could not be observed with the present statistics. The $B(M 1) / B(E 2)$ branching ratios for some of the observed bands are shown in Fig. 3. One can see an increase at spin around 20 for the bands D1, D3, and D4, which are well correlated with an increased single-particle alignment marking a change in the configuration. The $B(M 1) / B(E 2)$ values of all bands have a decreasing behavior at the highest spins. For the strongest pair of chiral bands, D5 and D5-chiral, we could extract the $B(M 1) / B(E 2)$ values for both bands, which are nearly identical within errors in the observed spin range.

Generally speaking, for the description of chiral rotations, three-dimensional tilted axis cranking covariant density functional theory (3D TAC-CDFT) should be adopted, which has been used to investigate the chiral modes of ${ }^{106} \mathrm{Rh}$ [42]. For the doublet bands built on the 4- and 6-quasiparticle configurations identified in ${ }^{136} \mathrm{Nd}$, the 3D TAC-CDFT calculations are very challenging. For simplicity, the observed five pairs of rotational bands are investigated within the framework of TAC-CDFT [43-45]. However, the chiral nature 


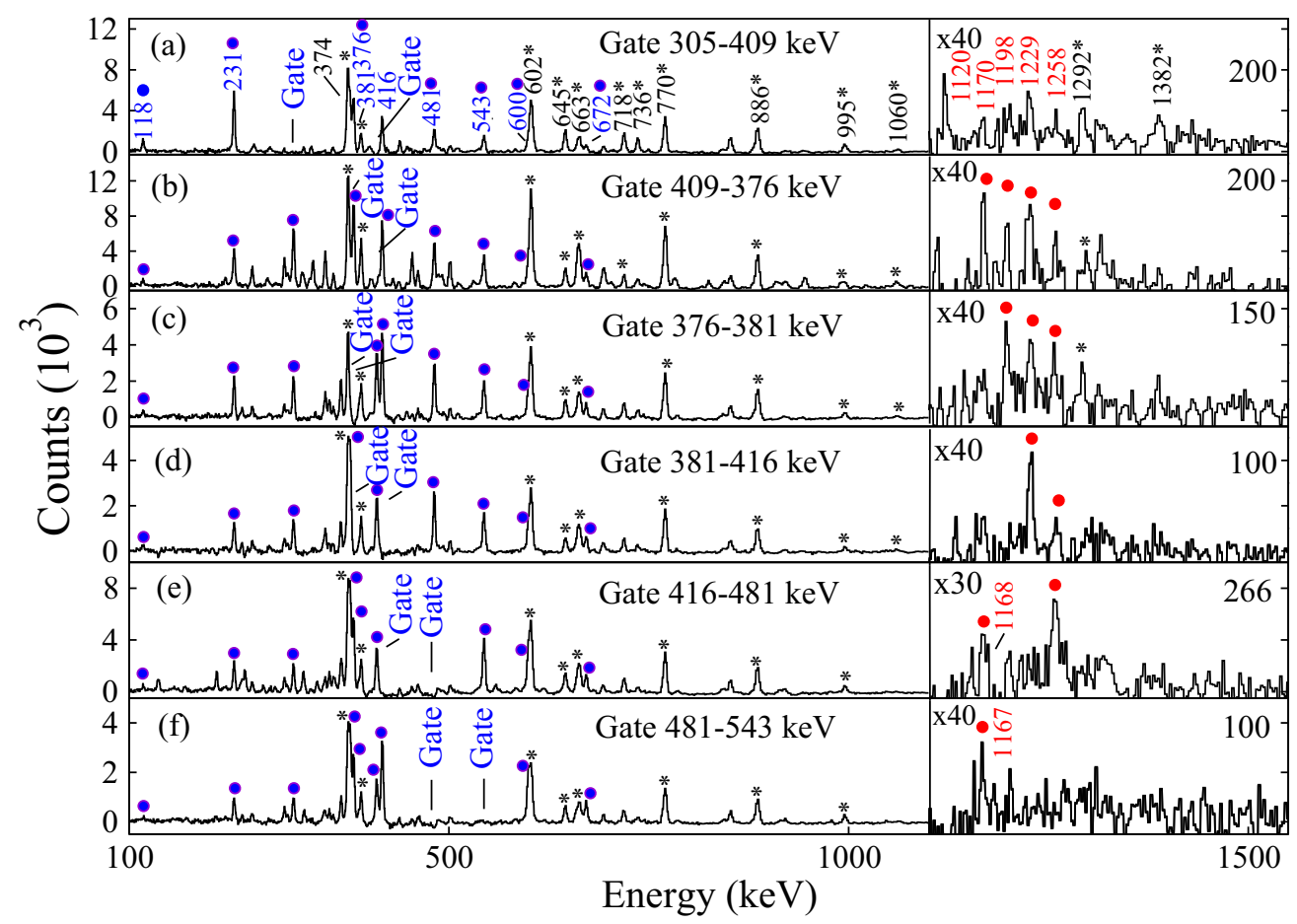

FIG. 2. Spectra constructed by double-gating on transitions of band $D 2$ which shows the connecting transitions of band $D 2$-c. The transitions marked with asterisks indicate low-lying transitions in ${ }^{136} \mathrm{Nd}$. The red lines show how the connecting transitions disappear when gating on successive higher-lying transitions of band $D 2$.

of the assigned configurations was tested by performing 3D TAC-CDFT calculations for one chiral pair as an example. The well-known relativistic density functional PC-PK1 [46] is adopted and the Dirac equation is solved in a 3D harmonic oscillator basis in Cartesian coordinates with 10 major shells which provide convergent results in TAC-CDFT calculations [45].

By minimizing the energy with respect to the triaxial deformation $\gamma$, both adiabatic and configuration-fixed constrained calculations similar to those in Ref. [18] were performed for various low-lying particle-hole excitations in ${ }^{136} \mathrm{Nd}$. Detailed results are shown in Table I. There are three positive-parity

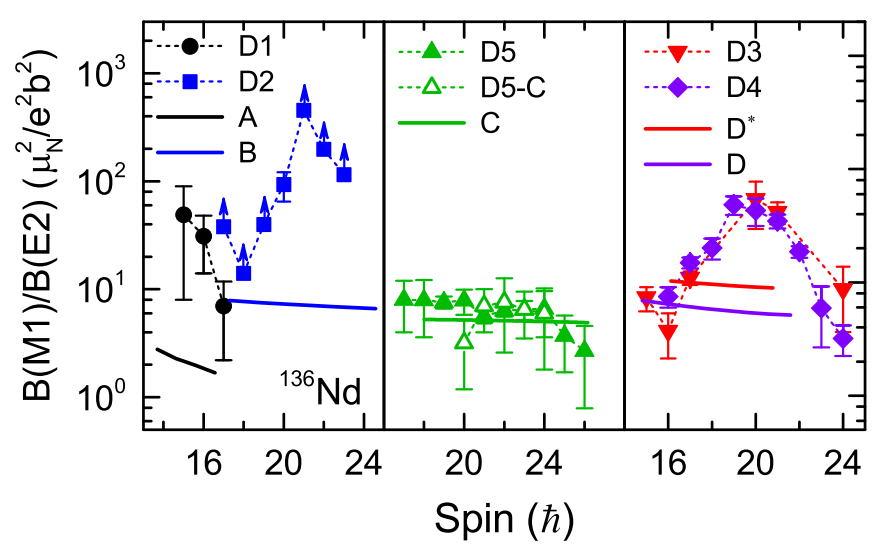

FIG. 3. Values of transition probabilities $B(M 1) / B(E 2)$ of ${ }^{136} \mathrm{Nd}$ calculated by TAC-CDFT, in comparison with experimental data (solid and open symbols). configurations (labeled A, B, and C) and five negativeparity configurations (labeled D, D*, E, F, and $\mathrm{H}$ ) which are candidates for the observed nearly degenerate partner bands. All these configurations with particle-hole excitations possess remarkable triaxial deformation, which is the typical feature for the chiral rotational bands. By considering the pairing correlations with separable pairing force [47,48], the changes of total energy and total angular momentum at the rotational frequency $0.2 \mathrm{MeV}$ are within $0.005 \%$ and $4.5 \%$ for configuration A. Therefore, pairing correlations can be safely neglected here. In the following, the configurations listed in Table I will be justified based on the quasiparticle alignments. The energy spectra, angular momenta, as well as the $B(M 1) / B(E 2)$ values of the observed doublet bands are also investigated within the TAC-CDFT framework for the assigned configurations.

Here, we take the bands D1 and D1-C as an example to show how the configurations are assigned. The quasiparticle alignments calculated by TAC-CDFT are shown in Fig. 4 in comparison with the experimental data. The experimental quasiparticle alignments are derived as in Ref. [49]. The parameters $\mathcal{J}_{0}=11 \hbar^{2} / \mathrm{MeV}$ and $\mathcal{J}_{1}=20 \hbar^{4} / \mathrm{MeV}^{3}$ have been adopted for the Harris formula $\mathcal{J}=\mathcal{J}_{0}+\mathcal{J}_{1} \omega^{2}$. It is clearly seen that the calculated results based on configuration A reproduce the experimental data of band D1 very well. Therefore, the configuration of band D1 is assigned as A, as shown in Table. I.

The assigned configuration $\mathrm{A}$ is further investigated by examining the calculated excitation energies of bands D1 and D1-C using the TAC-CDFT formalism, as shown in the left panels of Fig. 5. Note that the energy references for the 
TABLE I. Unpaired nucleon configurations labeled A-H and the corresponding parities, calculated by constrained CDFT. The deformation parameters $\beta$ and $\gamma$, and the excitation energies $E_{x}$ are also provided. It is noted that the letter $\mathrm{G}$ represents the ground state of ${ }^{136} \mathrm{Nd}$ and there are no nucleons resulting from the breaking of a Cooper pair for this configuration. $\Omega$ is the projection of the spin of the $d_{3 / 2}$ orbital on the quantization axis, while $\pi$ and $v$ indicate protons and neutrons, respectively. The configurations are given in terms of spherical single-particle orbitals.

\begin{tabular}{lccccc}
\hline \hline State & $E_{x}(\mathrm{MeV})$ & Parity & $\beta$ & $\gamma$ & Unpaired nucleons \\
\hline $\mathrm{G}$ & 0.000 & + & 0.24 & $27^{\circ}$ & - \\
$\mathrm{A}$ & 0.335 & + & 0.21 & $21^{\circ}$ & $\pi\left(h_{11 / 2}\right)^{1}\left(d_{5 / 2} g_{7 / 2}\right)^{-1} \otimes v\left(h_{11 / 2}\right)^{-1}\left(s_{1 / 2} d_{3 / 2}\right)^{-1}$ \\
$\mathrm{~B}$ & 3.419 & + & 0.22 & $19^{\circ}$ & $\pi\left(h_{11 / 2}\right)^{3}\left(d_{5 / 2} g_{7 / 2}\right)^{-1} \otimes v\left(h_{11 / 2}\right)^{-1}\left(s_{1 / 2} d_{3 / 2}\right)^{-1}$ \\
$\mathrm{C}$ & 3.704 & + & 0.26 & $23^{\circ}$ & $\pi\left(h_{11 / 2}\right)^{2}\left(d_{5 / 2} g_{7 / 2}\right)^{-2} \otimes v\left(h_{11 / 2}\right)^{-1}\left(f_{7 / 2} h_{9 / 2}\right)^{1}$ \\
$\mathrm{D}$ & 1.173 & - & 0.22 & $19^{\circ}$ & $\pi\left(h_{11 / 2}\right)^{2} \otimes v\left(h_{11 / 2}\right)^{-1}\left(s_{1 / 2} d_{3 / 2}\right)^{-1}\left(\Omega \sim+\frac{3}{2}\right)$ \\
$\mathrm{D}^{*}$ & 1.346 & - & 0.21 & $22^{\circ}$ & $\pi\left(h_{11 / 2}\right)^{2} \otimes v\left(h_{11 / 2}\right)^{-1}\left(s_{1 / 2} d_{3 / 2}\right)^{-1}\left(\Omega \sim-\frac{3}{2}\right)$ \\
$\mathrm{E}$ & 1.937 & - & 0.21 & $23^{\circ}$ & $\pi\left(h_{11 / 2}\right)^{2}\left(d_{5 / 2} g_{7 / 2}\right)^{-2} \otimes v\left(h_{11 / 2}\right)^{-1}\left(s_{1 / 2} d_{3 / 2}\right)^{-1}$ \\
$\mathrm{~F}$ & 2.778 & - & 0.20 & $35^{\circ}$ & $\pi\left(h_{11 / 2}\right)^{1}\left(d_{5 / 2} g_{7 / 2}\right)^{-1} \otimes v\left(h_{11 / 2}\right)^{-2}$ \\
$\mathrm{H}$ & 3.494 & - & 0.20 & $37^{\circ}$ & $\pi\left(h_{11 / 2}\right)^{1}\left(d_{5 / 2} g_{7 / 2}\right)^{-3} \otimes v\left(h_{11 / 2}\right)^{-2}$ \\
\hline \hline
\end{tabular}

positive- and negative-parity calculated bands are taken as those of band D1 at $I=11 \hbar$ and of band D4 at $I=15 \hbar$, respectively. It is found that the theoretical results based on configuration A agree with experimental data of band D1 satisfactorily, and the configuration assignment to band D1 is validated. Meanwhile, no other configuration can be found to reproduce the results of D1-C, which indicates that bands $\mathrm{D} 1$ and D1-C are based on the same intrinsic state. Therefore one can safely identify D1 and D1-C as good candidates for chiral partner bands.

Similarly, one can conclude that D2 and D2-C, D5 and D5C, D3 and D3-C, a well as D4 and D4-C are candidates of chiral doublets bands built on the same intrinsic configurations $\mathrm{B}, \mathrm{C}, \mathrm{D}^{*}$, and D, respectively. It is noted that the back-bending phenomenon observed in bands D2, D3, and D4 can be due to crossing with 8-, 6-, and 6-qp configurations, respectively, which is beyond the current TAC-CDFT calculation.

The $B(M 1) / B(E 2)$ values of the five pairs of chiral bands have also been studied self-consistently, as shown in Fig. 3. The calculated $B(M 1) / B(E 2)$ values based on configurations $A$ and $B$ underestimate the experimental data of bands D1 and $\mathrm{D} 2$, while the theoretical $B(M 1) / B(E 2)$ values of the assigned

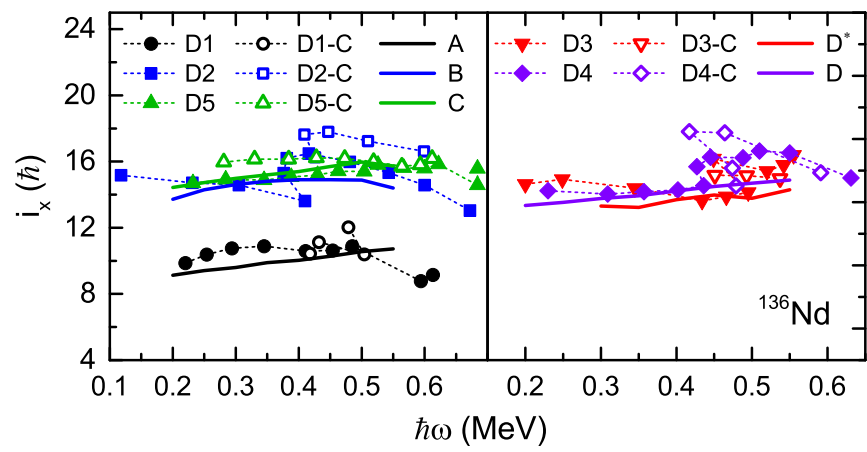

FIG. 4. Quasiparticle alignments calculated by TAC-CDFT for the positive-parity (left panel) and negative-parity (right panel) chiral rotational bands of ${ }^{136} \mathrm{Nd}$. Solid and open circles with the same color represent experimental data of one pair of nearly degenerate bands, and different lines denote the theoretical results based on different configurations. configuration are in good agreement with the experimental data of band D5 over all the observed spin range. As for bands D3 and D4, a satisfactory agreement with experimental is only present in the low- and high-spin parts. An abrupt increase of $B(M 1) / B(E 2)$ values in bands D3 and D4 is observed near back-bending and cannot be reproduced by the calculations. As mentioned above, a back-bending also exists in band D2, which induces an abrupt increase of the $B(M 1) / B(E 2)$ values. Note that although the absolute $B(M 1) / B(E 2)$ values for bands D1 and D2 are not reproduced, the relative differences agree with the experimental data well. A better agreement over the entire observed spin range will hopefully be obtained in the near future with extensive theoretical calculations taking into account the possible change of configuration along the bands and the configuration mixing.

Finally, to give more theoretical support for the observed chiral rotation in ${ }^{136} \mathrm{Nd}$, we also performed challenging calculations based on 3D TAC-CDFT. The $\mathrm{D}^{*}$ configuration corresponding to band D3 was calculated as an example. The orientation angle $\phi$ for the total angular momentum of band D3 calculated with 3D TAC-CDFT is shown in Fig. 6.

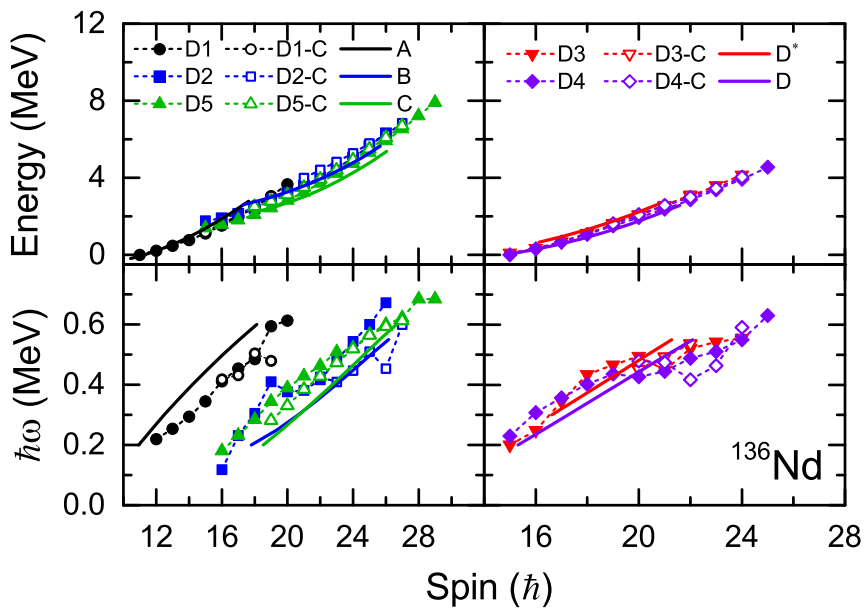

FIG. 5. Excitation energies and $\hbar \omega$ vs $I$ calculated by TAC-CDFT for the positive (left panel) and negative (right panel) chiral rotational bands of ${ }^{136} \mathrm{Nd}$. 


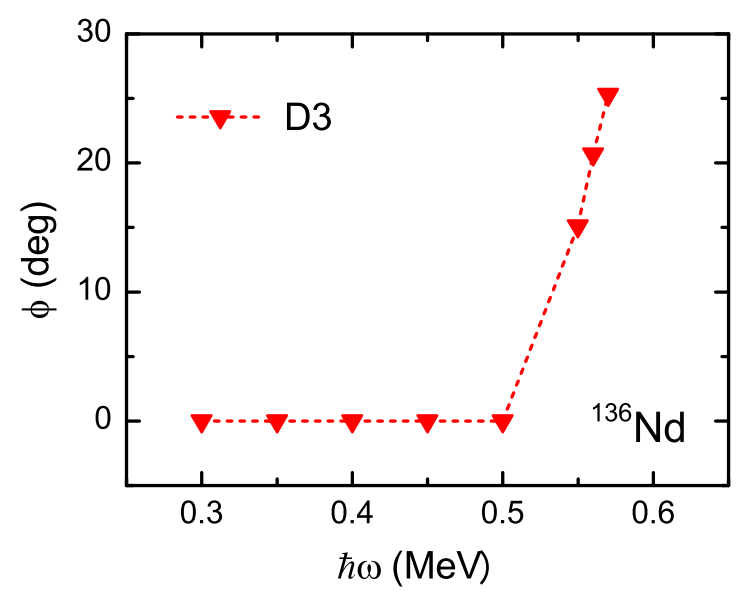

FIG. 6. Evolution of the azimuth angle $\phi$ as a function of rotational frequency, for the total angular momentum of the configuration D* assigned to band D3, calculated by 3D TAC-CDFT.

It is found that the azimuth angle $\phi$ for band D3 vanishes at low rotational frequencies, providing a planar solution. Above the critical rotational frequency $0.5 \mathrm{MeV}$, a nonzero angle $\phi$ appears, corresponding to an aplanar solution, namely, chiral rotation, for the selected configuration, namely, $D^{*}$. The resulting excitation energies and total angular momenta are well reproduced by the present 3D TAC-CDFT calculations. We find aplanar solutions in band D3 which provides very strong support for the existence of chiral rotation. Moreover, the effects of $3 \mathrm{D}$ rotation on $B(M 1) / B(E 2)$ values, the total angular momentum, and excitation energies are marginal.

Summarizing, five pairs of nearly degenerate rotational bands were identified in ${ }^{136} \mathrm{Nd}$, one of which has a clear chiral character. It is the first time that chiral bands are observed in an even-even nucleus at high spins. The other four doublet bands, which are weakly populated, have no measured $B(M 1) / B(E 2)$ values, but the calculated assigned configurations show chiral geometry. This set of five nearly degenerate bands is the largest observed in a single nucleus until now. The observed bands were discussed using the TAC-CDFT, 3D TAC-CDFT, and MQ-PRM models, which reveal the chiral nature in the assigned configurations. Further theoretical calculations to investigate in detail to what extent the chiral geometry of the observed bands is realized are in progress.

This work has been supported by the Academy of Finland under the Finnish Centre of Excellence Programme (20122017) and by the EU 7th Framework Programme Project No. 262010 (ENSAR). The use of germanium detectors from the GAMMAPOOL is acknowledged. This work was supported in part by the GINOP-2.3.3-15-2016-00034, National Research, Development and Innovation Office NKFIH, Contract No. PD 124717, the Polish National Science Centre (NCN) Grant No. 2013/10/M/ST2/00427, the Swedish Research Council under Grant No. 621-2014-5558, and the Chinese Major State 973 Program No. 2013CB834400, National Natural Science Foundation of China (Grants No. 11335002, No. 11375015, No. 11461141002, and No. 11621131001).
[1] S. Frauendorf and J. Meng, Nucl. Phys. A 617, 131 (1997).

[2] S. Frauendorf, Rev. Mod. Phys. 73, 463 (2001).

[3] J. Meng and S. Q. Zhang, J. Phys. G. 37, 064025 (2010).

[4] J. Meng and P. Zhao, Phys. Scr. 91, 053008 (2016).

[5] S. Mukhopadhyay, D. Almehed, U. Garg, S. Frauendorf, T. Li, P. V. M. Rao, X. Wang, S. S. Ghughre, M. P. Carpenter, S. Gros, A. Hecht, R.V.F. Janssens, F. G. Kondev, T. Lauritsen, D. Seweryniak, and S. Zhu, Phys. Rev. Lett. 99, 172501 (2007).

[6] S. Y. Wang, B. Qi, L. Liu, S. Q. Zhang, H. Hua, X. Q. Li, Y. Y. Chen, L. H. Zhu, J. Meng, S. M. Wyngaardt, P. Papka, T. T. Ibrahim, R. A. Bark, P. Datta, E. A. Lawrie, J. J. Lawrie, S. N. T. Majola, P. L. Masiteng, S. M. Mullins, J. Geral, G. Kalinka, J. Molnár, B. M. Nyakó, J. Timár, K. Juhász, and R. Schwengner, Phys. Lett. B 703, 40 (2011).

[7] C. Liu, S. Y. Wang, R. A. Bark, S. Q. Zhang, J. Meng, B. Qi, P. Jones, S. M. Wyngaardt, J. Zhao, C. Xu, S. G. Zhou, S. Wang, D. P. Sun, L. Liu, Z. Q. Li, N. B. Zhang, H. Jia, X. Q. Li, H. Hua, Q. B. Chen, Z. G. Xiao, H. J. Li, L. H. Zhu, T. D. Bucher, T. Dinoko, J. Easton, K. Juhasz, A. Kamblawe, E. Khaleel, N. Khumalo, E. A. Lawrie, J. J. Lawrie, S.N.T. Majola, S. M. Mullins, S. Murray, J. Ndayishimye, D. Negi, S. P. Noncolela, S. S. Ntshangase, B. M. Nyako, J. N. Orce, P. Papka, J. F. Sharpey-Schafer, O. Shirinda, P. Sithole, M. A. Stankiewicz, and M. Wiedeking, Phys. Rev. Lett. 116, 112501 (2016).

[8] C. M. Petrache, D. Bazzacco, S. Lunardi, C. Rossi Alvarez, G. de Angelis, M. De Poli, D. Bucurescu, C. A. Ur, P. B. Semmes, and R. Wyss, Nucl. Phys. A 597, 106 (1996).
[9] K. Starosta, T. Koike, C. J. Chiara, D. B. Fossan, D. R. LaFosse, A. A. Hecht, C. W. Beausang, M. A. Caprio, J. R. Cooper, R. Krücken, J. R. Novak, N. V. Zamfir, K. E. Zyromski, D. J. Hartley, D. Balabanski, J.-y. Zhang, S. Frauendorf, and V. I. Dimitrov, Phys. Rev. Lett. 86, 971 (2001).

[10] A. A. Hecht, C. W. Beausang, K. E. Zyromski, D. L. Balabanski, C. J. Barton, M. A. Caprio, R. F. Casten, J. R. Cooper, D. J. Hartley, R. Krücken, D. Meyer, H. Newman, J. R. Novak, E. S. Paul, N. Pietralla, A. Wolf, N. V. Zamfir, J.-y. Zhang, and F. Dönau, Phys. Rev. C 63, 051302(R) (2001).

[11] T. Koike, K. Starosta, C. J. Chiara, D. B. Fossan, and D. R. LaFosse, Phys. Rev. C 63, 061304(R) (2001).

[12] R. A. Bark, A. M. Baxter, A. P. Byrne, G. D. Dracoulis, T. Kibédi, T. R. McGoram, and S. M. Mullins, Nucl. Phys. A 691, 577 (2001).

[13] T. Koike, K. Starosta, C. J. Chiara, D. B. Fossan, and D. R. LaFosse, Phys. Rev. C 67, 044319 (2003).

[14] C. Vaman, D. B. Fossan, T. Koike, K. Starosta, I. Y. Lee, and A. O. Macchiavelli, Phys. Rev. Lett. 92, 032501 (2004).

[15] P. Joshi, D. G. Jenkins, P. M. Raddon, A. J. Simons, R. Wadsworth, A. R. Wilkinson, D. B. Fossan, T. Koike, K. Starosta, C. Vaman, A. Algora, E. S. Paul, G. Rainovski, A. Gizon, J. Gizon, P. Bednarczyk, D. Curien, G. Duchêne, and J. N. Scheurer, Phys. Lett. B 595, 135 (2004).

[16] E. Grodner, J. Srebrny, A. A. Pasternak, I. Zalewska, T. Morek, C. Droste, J. Mierzejewski, M. Kowalczyk, J. Kownacki, M. Kisieliński, S. G. Rohoziński, T. Koike, K. Starosta, 
A. Kordyasz, P. J. Napiorkowski, M. Wolińska-Cichocka, E. Ruchowska, W. Płóciennik, and J. Perkowski, Phys. Rev. Lett. 97, 172501 (2006).

[17] S. Zhu, U. Garg, B. K. Nayak, S. S. Ghugre, N. S. Pattabiraman, D. B. Fossan, T. Koike, K. Starosta, C. Vaman, R.V.F. Janssens, R. S. Chakrawarthy, M. Whitehead, A. O. Macchiavelli, and S. Frauendorf, Phys. Rev. Lett. 91, 132501 (2003).

[18] J. Meng, J. Peng, S. Q. Zhang, and S.-G. Zhou, Phys. Rev. C 73, 037303 (2006).

[19] J. Peng, H. Sagawa, S. Q. Zhang, J. M. Yao, Y. Zhang, and J. Meng, Phys. Rev. C 77, 024309 (2008).

[20] J. M. Yao, B. Qi, S. Q. Zhang, J. Peng, S. Y. Wang, and J. Meng, Phys. Rev. C 79, 067302 (2009).

[21] J. Li, S. Q. Zhang, and J. Meng, Phys. Rev. C 83, 037301 (2011).

[22] C. Droste, S. G. Rohoziński, K. Starosta, L. Próchniak, and E. Grodner, Eur. Phys. J. A 42, 79 (2009).

[23] I. Kuti, Q. B. Chen, J. Timár, D. Sohler, S. Q.Zhang, Z. H. Zhang, P. W. Zhao, J. Meng, K. Starosta, T. Koike, E. S. Paul, D. B. Fossan, and C. Vaman, Phys. Rev. Lett. 113, 032501 (2014).

[24] A. D. Ayangeakaa, U. Garg, M. D. Anthony, S. Frauendorf, J. T. Matta, B. K. Nayak, D. Patel, Q. B. Chen, S. Q. Zhang, P. W. Zhao, B. Qi, J. Meng, R.V.F. Janssens, M. P. Carpenter, C. J. Chiara, F. G. Kondev, T. Lauritsen, D. Seweryniak, S. Zhu, S. S. Ghugre, and R. Palit, Phys. Rev. Lett. 110, 172504 (2013).

[25] E. Mergel, C. M. Petrache, G. Lo Bianco, H. Hübel, J. Domscheit, D. Rossbach, G. Schönwasser, N. Nenoff, A. Neusser, A. Görgen, F. Becker, E. Bouchez, M. Houry, A. Hürstel, Y. Le Coz, R. Lucas, Ch. Theisen, W. Korten, A. Bracco, N. Blasi, F. Camera, S. Leoni, F. Hannachi, A. Lopez-Martens, M. Rejmund, D. Gassmann, P. Reiter, P. G. Thirolf, A. Astier, N. Buforn, M. Meyer, N. Redon, and O. Stezowski, Eur. Phys. J. A 15, 417 (2002).

[26] S. Mukhopadhyay, D. Almehed, U. Garg, S. Frauendorf, T. Li, P. V. M. Rao, X. Wang, S. S. Ghugre, M. P. Carpenter, S. Gros, A. Hecht, R.V.F. Janssens, F. G. Kondev, T. Lauritsen, D. Seweryniak, and S. Zhu, Phys. Rev. C 78, 034311 (2008).

[27] Y. X. Luo, S. J. Zhu, J. H. Hamilton, J. O. Rasmussen, A. V. Ramayya, C. Goodin, K. Li, J. K. Hwang, D. Almehed, S. Frauendorf, V. Dimitrov, Jing-ye Zhang, X. L. Che, Z. Jang, I. Stefanescu, A. Gelberg, G. M. Ter-Akopian, A. V. Daniel, M. A. Stoyer, R. Donangelo, J. D. Cole, and N. J. Stone, Phys. Lett. B 670, 307 (2009).

[28] E. S. Paul, C. W. Beausang, D. B. Fossan, R. Ma, W. F. Piel, Jr., P. K. Weng, and N. Xu, Phys. Rev. C 36, 153 (1987).

[29] J. Billowes, K. P. Lieb, J. W. Noé, W. F. Piel, Jr., S. L. Rolston, G. D. Sprouse, O. C. Kistner, and F. Christancho, Phys. Rev. C 36, 974 (1987).

[30] C. M. Petrache, D. Bazzacco, S. Lunardi, C. Rossi Alvarez, R. Venturelli, D. Bucurescu, C. A. Ur, D. De Acuna, G. Maron, D. R. Napoli, N. H. Medina, J. R. B. Oliveira, and R. Wyss, Phys. Lett. B 373, 275 (1996).

[31] C. M. Petrache, Y. Sun, D. Bazzacco, S. Lunardi, C. R. Alvarez, R. Venturelli, D. De Acuña, G. Maron, M. N. Rao, Z. Podolyák, and J. R. B. Oliveira, Phys. Rev. C 53, R2581(R) (1996).
[32] S. Perries, A. Astier, L. Ducroux, M. Meyer, N. Redon, C. M. Petrache, D. Bazzacco, G. Falconi, S. Lunardi, M. Lunardon, C. RossiAlvarez, C. A. Ur, R. Venturelli, G. Viesti, I. Deloncle, M. G. Porquet, G. deAngelis, M. dePoli, C. Fahlander, E. Farnea, D. Foltescu, A. Gadea, D. R. Napoli, Z. Podolyak, A. Bracco, S. Frattini, S. Leoni, B. Cederwall, A. Johnson, and R. A. Wyss, Phys. Rev. C 60, 064313 (1999).

[33] R. Saito, N. Saito, K. Starosta, J. Beller, N. Pietralla, H. J. Wollersheim, D. L. Balabanski, A. Banu, R. A. Bark, T. Beck, F. Becker, P. Bednarczyk, K.-H. Behr, G. Benzoni, P. G. Bizzeti, C. Boiano, A. Bracco, S. Brambilla, A. Brünle, A. Bürger, L. Caceres, F. Camera, F. C. L. Crespi, P. Doornenbal, A. B. Garnsworthy, H. Geissel, J. Gerl, M. Górska, J. Grebosza, G. Hagemann, J. Jolie, M. Kavatsyuk, O. Kavatsyuk, T. Koike, I. Kojouharov, N. Kurz, J. Leske, G. Lo Bianco, A. Maj, S. Mallion, S. Mandal, M. Maliage, T. Otsuka, C. M. Petrache, Zs. Podolyak, W. Prokopowicz, G. Rainovski, P. Reiter, A. Richard, H. Schaffner, S. Schielke, G. Sletten, N. J. Thompson, D. Tonev, J. Walker, N. Warr, O. Wieland, and Q. Zhong, Phys. Lett. B 669, 19 (2008).

[34] B. F. Lv et al. (unpublished).

[35] P. Rahkila, Nucl. Instrum. Methods Phys. Res. A 595, 637 (2008).

[36] D. Radford, Nucl. Instrum. Methods Phys. Res. A 361, 297 (1995).

[37] D. Radford, Nucl. Instrum. Methods Phys. Res. A 361, 306 (1995)

[38] A. Krämer-Flecken, T. Morek, R. M. Lieder, W. Gast, G. Hebbinghaus, H. M. Jäger, and W. Urban, Nucl. Instrum. Methods Phys. Res. A 275, 333 (1989).

[39] C. J. Chiara, M. Devlin, E. Ideguchi, D. R. LaFosse, F. Lerma, W. Reviol, S. K. Ryu, D. G. Sarantites, O. L. Pechenaya, C. Baktash, A. Galindo-Uribarri, M. P. Carpenter, R.V.F. Janssens, T. Lauritsen, C. J. Lister, P. Reiter, D. Seweryniak, P. Fallon, A. Görgen, A. O. Macchiavelli, D. Rudolph, G. Stoitcheva, and W. E. Ormand, Phys. Rev. C 75, 054305 (2007).

[40] V. Iacob and G. Duchene, Nucl. Instrum. Methods Phys. Res. A 399, 57 (1997).

[41] K. Starosta, T. Morek, Ch. Droste, S. G. Rohozinski, J. Srebrny, A. Wierzchucka, M. Bergström, B. Herskind, E. Melby, T. Czosnyka, and P. J. Napiorkowski, Nucl. Instrum. Methods Phys. Res. A 423, 16 (1999).

[42] P. Zhao, Phys. Lett. B 773, 1 (2017).

[43] J. Peng, J. Meng, P. Ring, and S. Q. Zhang, Phys. Rev. C 78, 024313 (2008).

[44] P. W. Zhao, S. Q. Zhang, J. Peng, H. Z. Liang, P. Ring, and J. Meng, Phys. Lett. B 699, 181 (2011).

[45] J. Meng, J. Peng, S. Q. Zhang, and P. W. Zhao, Front. Phys. 8, 55 (2013).

[46] P. W. Zhao, Z. P. Li, J. M. Yao, and J. Meng, Phys. Rev. C 82, 054319 (2010).

[47] P. W. Zhao, S. Q. Zhang, and J. Meng, Phys. Rev. C 92, 034319 (2015)

[48] Y. K. Wang, Phys. Rev. C 96, 054324 (2017).

[49] R. Bengtsson and S. Frauendorf, Nucl. Phys. A 327, 139 (1979). 